\title{
Photogrammetric Assessment of Flexure Induced Cracking of Reinforced Concrete Beams under Service Loads
}

Pease, Bradley Justin; Geiker, Mette Rica; Stang, Henrik; Weiss, Jason

Publication date:

2006

Link back to DTU Orbit

Citation (APA):

Pease, B. J., Geiker, M. R., Stang, H., \& Weiss, J. (2006). Photogrammetric Assessment of Flexure Induced Cracking of Reinforced Concrete Beams under Service Loads.

\section{General rights}

Copyright and moral rights for the publications made accessible in the public portal are retained by the authors and/or other copyright owners and it is a condition of accessing publications that users recognise and abide by the legal requirements associated with these rights.

- Users may download and print one copy of any publication from the public portal for the purpose of private study or research.

- You may not further distribute the material or use it for any profit-making activity or commercial gain

- You may freely distribute the URL identifying the publication in the public portal

If you believe that this document breaches copyright please contact us providing details, and we will remove access to the work immediately and investigate your claim. 


\title{
Photogrammetric Assessment of
} Flexural Induced Cracking in

Reinforced Concrete Beams under

\section{Service Loads}

\author{
Brad Pease, Mette Geiker, \\ Henrik Stang, and Jason Weiss
}

$2^{\mathrm{ND}}$ International RILEM Symposium,

September 11-13, 2006 


\title{
Outline of Today's Talk
}

- Sample Geometry

- Three-Dimensional Photogrammetry Technique

- Test Results

- Conclusions
- Crack Geometry
- Crack Measurements

- Conclusions

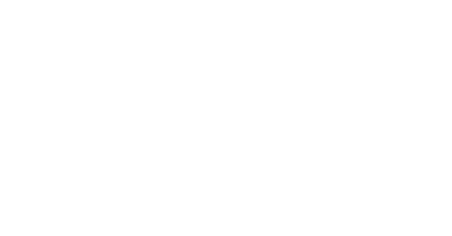

\section{- Motivation \& Goal
- Experimental Investigation \\ - Motivation \& Goal
- Experimental Investigation \\ igation}

.

GOROIUSIORS

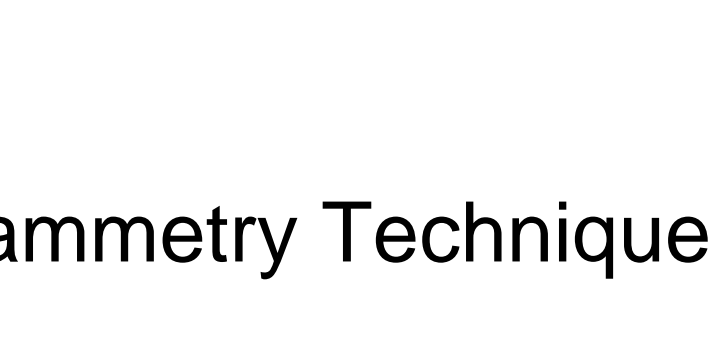

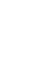

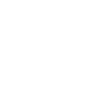

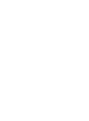

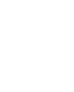

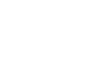

- Three-Dimensional Photogrammetry Technique

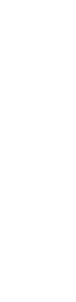

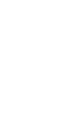

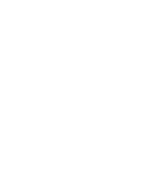

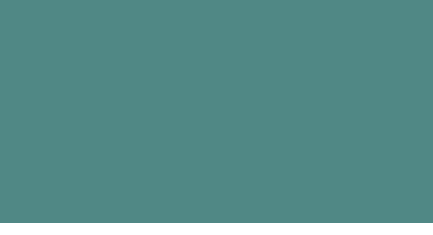


- Motivation

- Cracking in concrete affects ingress and corrosion (i.e. service life)

- Cracking in reinforced concrete is complicated by slip and separation at reinforcing bar

- Goal

- Quantify crack geometry in reinforced concrete beam during loading 
Reinforced beams
$(150 \times 150 \times 600 \mathrm{~mm})$ Reinforced beams
$(150 \times 150 \times 600 \mathrm{~mm})$

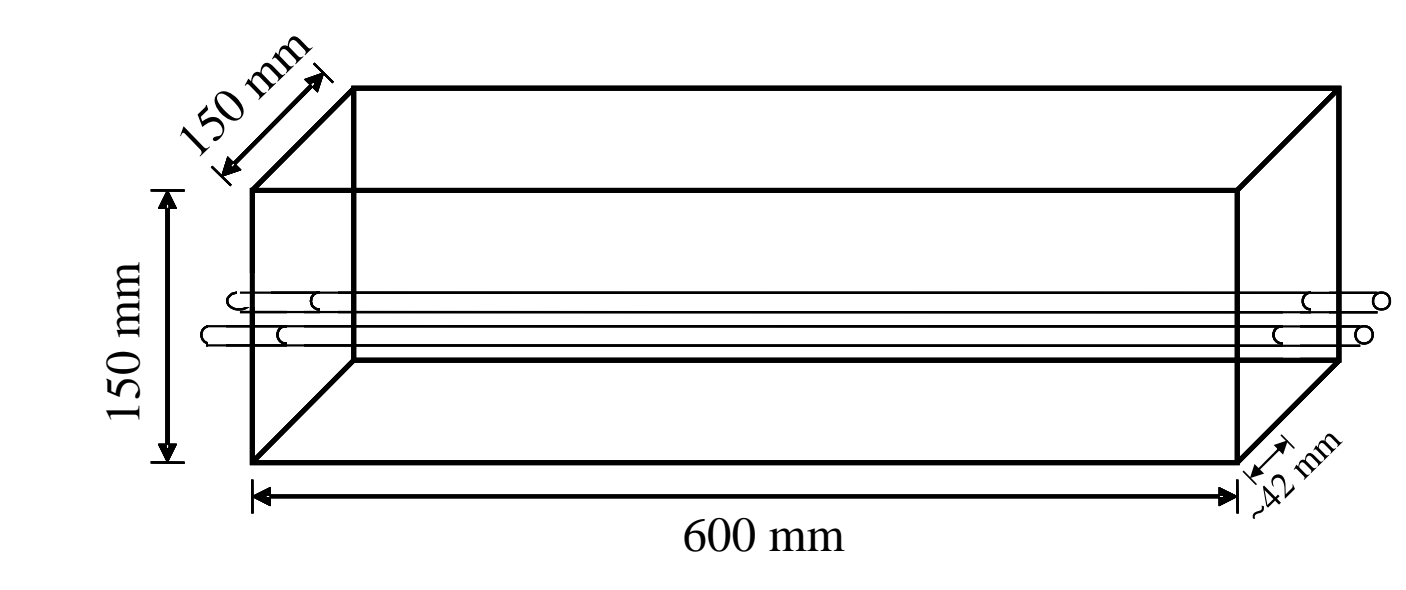

\section{Experimental Observation of Cracks: Sample
Geometry and Loading \\ Experimental Observation of Crack
Geometry and Loading

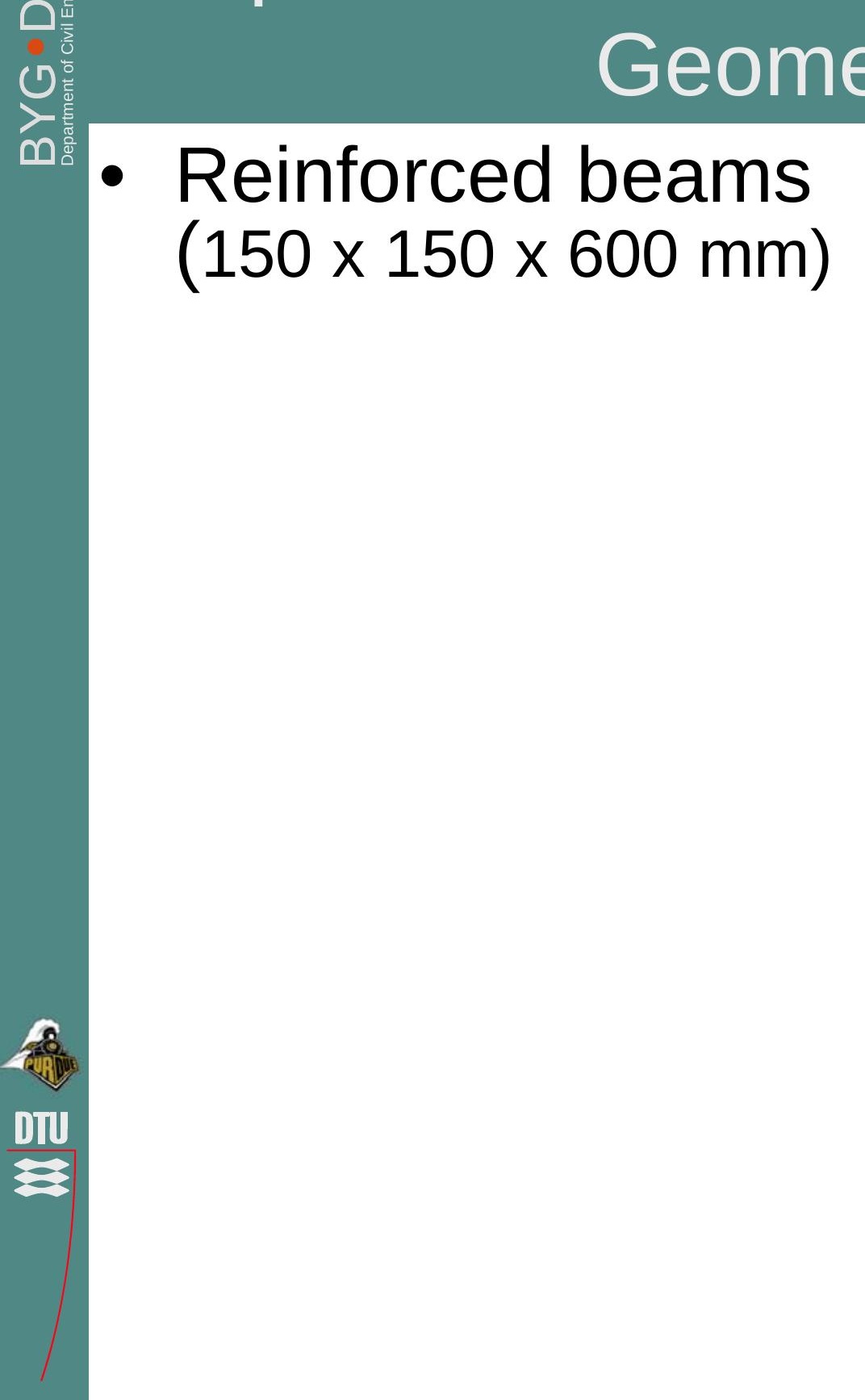


Reinforced beams
$(150 \times 150 \times 600 \mathrm{~mm})$ Reinforced beams
$(150 \times 150 \times 600 \mathrm{~mm})$

- Face of concrete removed

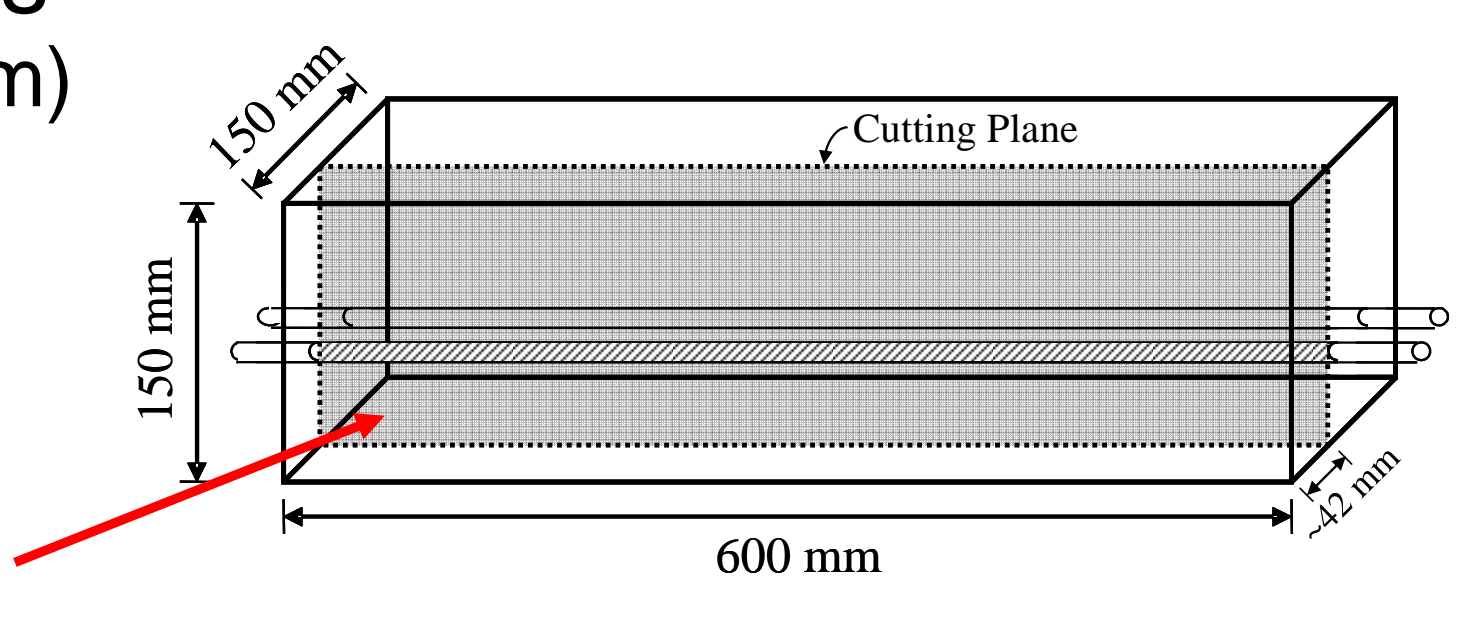
- Exposed
reinforcem
aggregate reinforcement and Exposed
reinforcem
aggregate

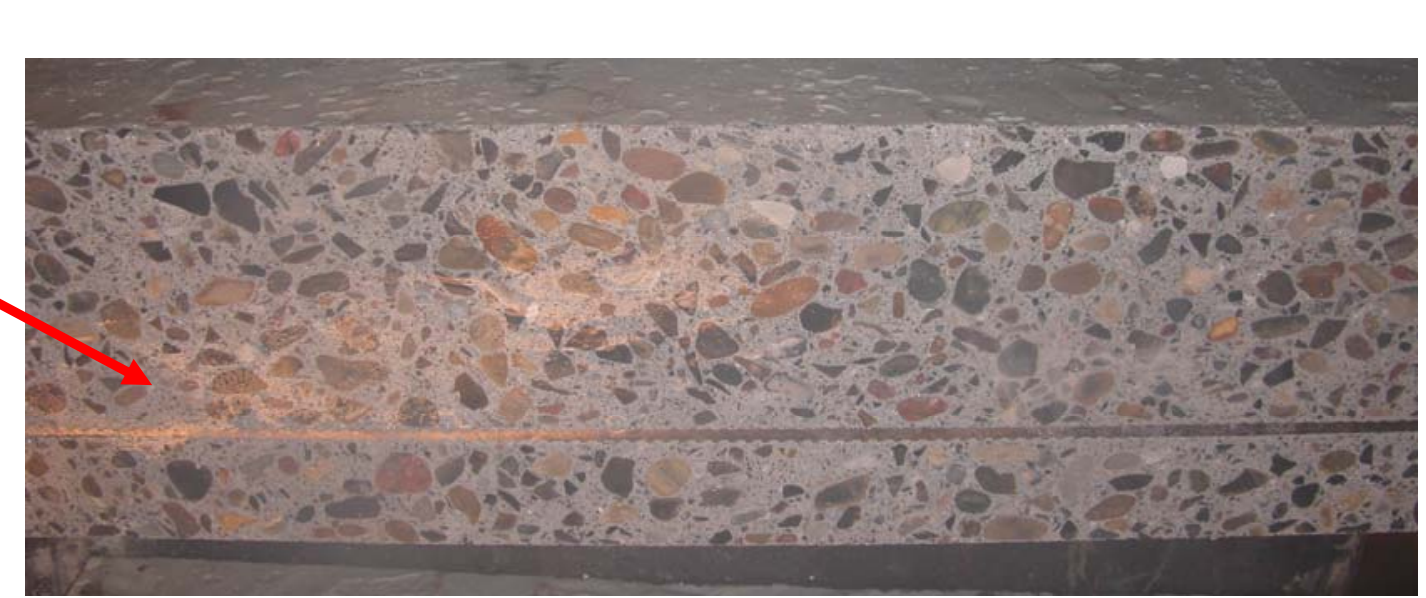

\section{Experimental Observation of Cracks: Sample \\ Geometry and Loading \\ (1)} Exposed
reinforcem
aggregate

\section{d}
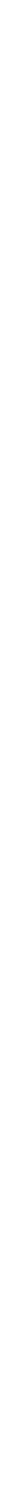
- Reinforced beams $(150 \times 150 \times 600 \mathrm{~mm})$ Face of concrete
removed

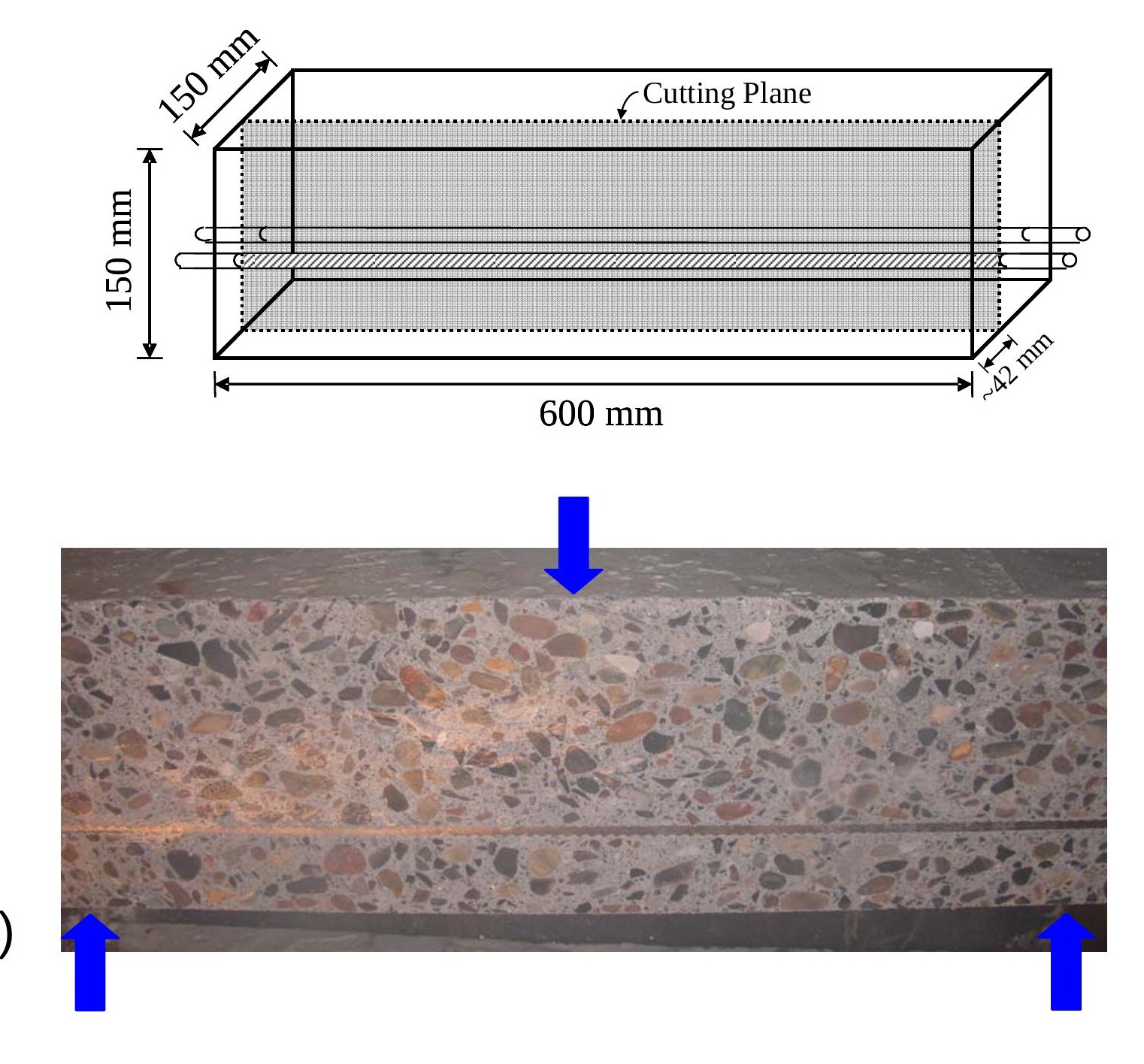
Exposed
reinforcement and
aggregate
3-Point bending
- 1.0 and 1.8 times
estimated cracking
load (13 and $25.2 \mathrm{kN}$ )
$-35 \mathrm{kN}$

Exposed
reinforcement and
aggregate
3-Point bending
-1.0 and 1.8 times
estimated cracking
load (13 and $25.2 \mathrm{kN})$
$-35 \mathrm{kN}$

Exposed
reinforcement and
aggregate
3-Point bending
- 1.0 and 1.8 times
estimated cracking
load (13 and $25.2 \mathrm{kN})$
- $35 \mathrm{kN}$

Exposed
reinforcement and
aggregate
3-Point bending
- 1.0 and 1.8 times
estimated cracking
load (13 and $25.2 \mathrm{kN}$ )
- $35 \mathrm{kN}$

Exposed
reinforcement and
aggregate
3-Point bending
- 1.0 and 1.8 times
$\quad$ estimated cracking
load (13 and $25.2 \mathrm{kN})$
- $35 \mathrm{kN}$

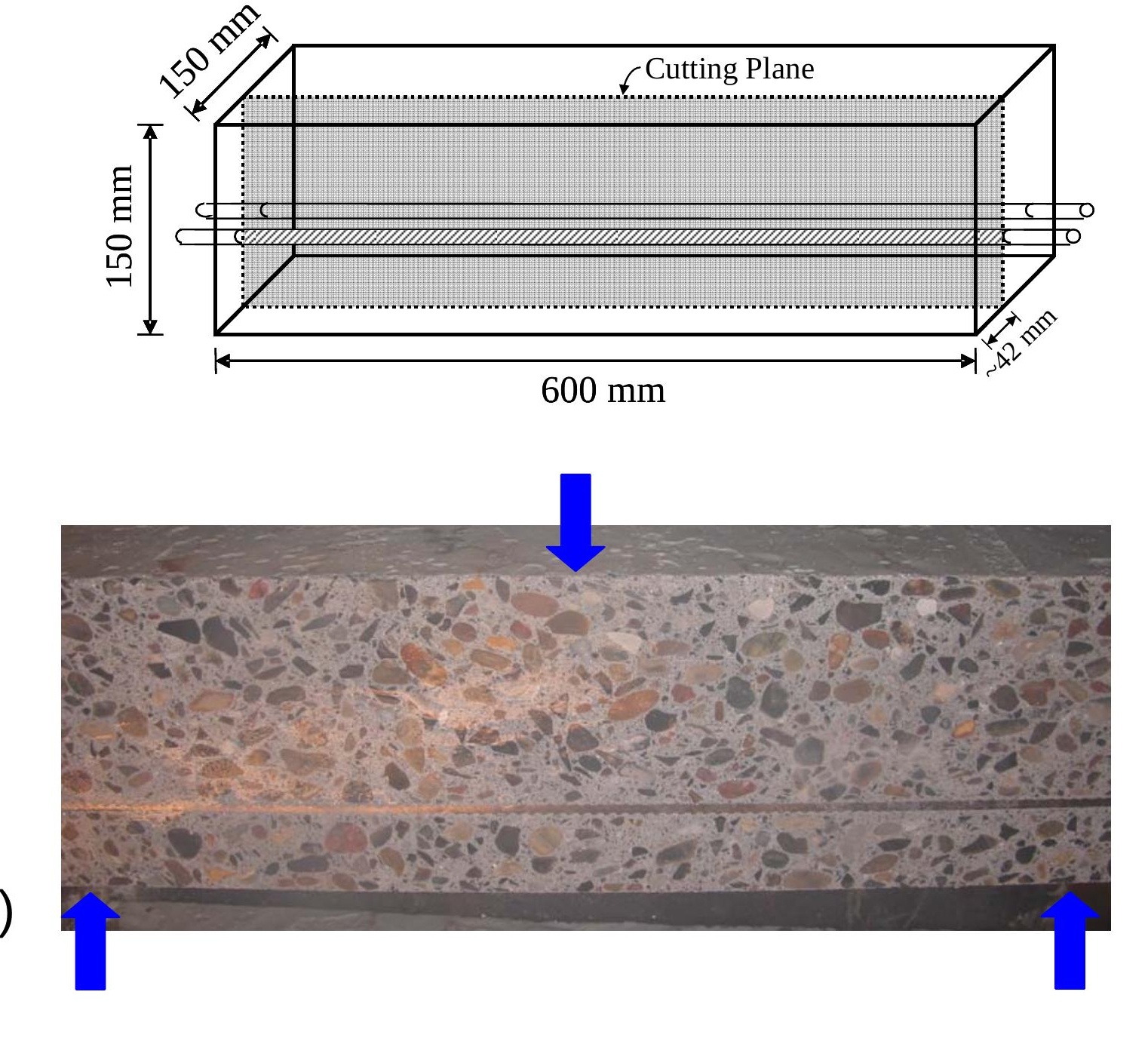

\section{금 Experimental Observation of Cracks: Sample \\ Geometry and Loading \\ C}

(n)
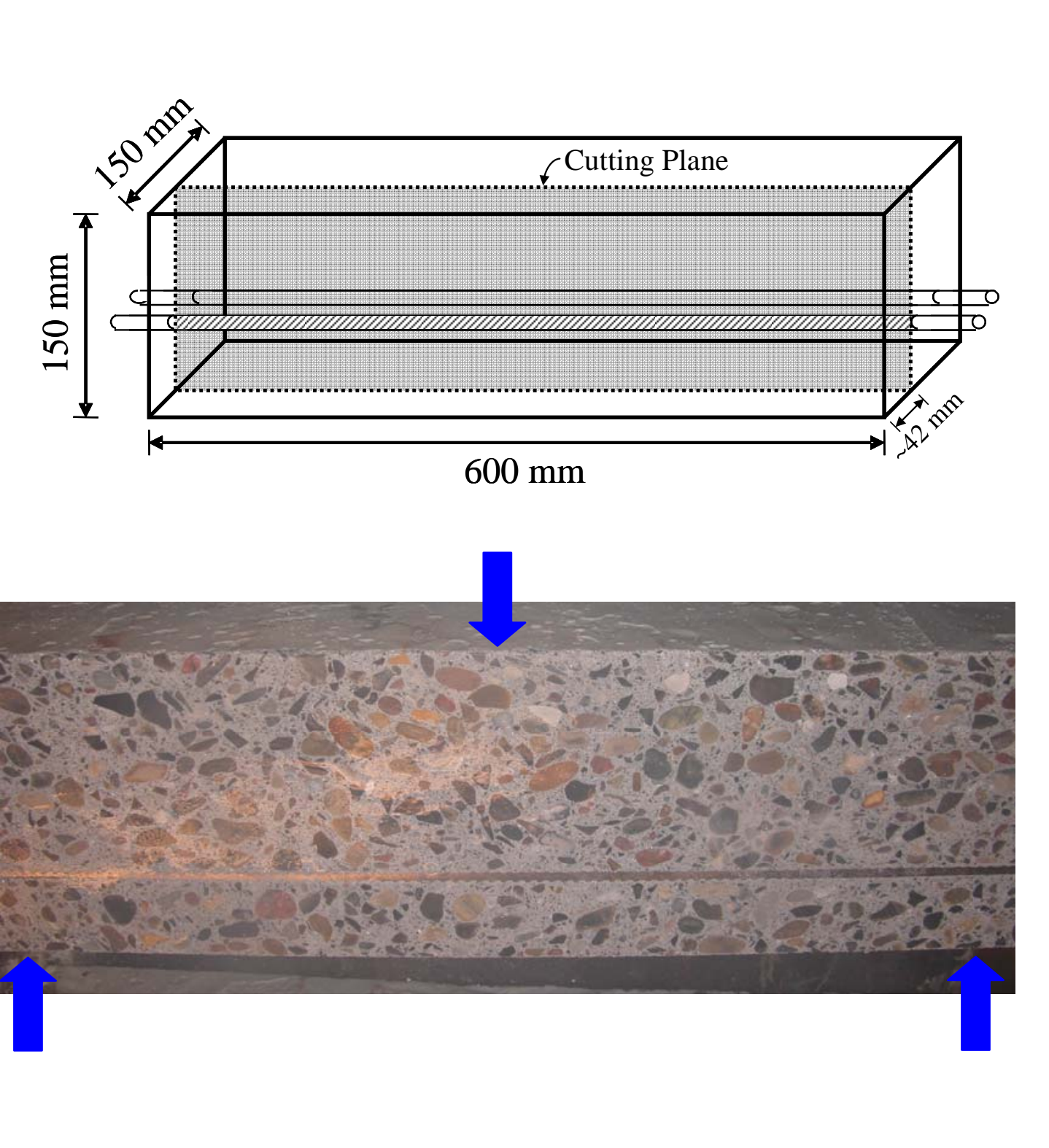

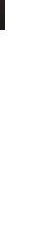




\section{3-D Photogrammetry}

- Photogrammetry quantifies cracking during loading

- Two digital cameras placed equidistant from sample, focused on same point collect images

- Images separated into a mesh by software

- Individual mesh box = facet
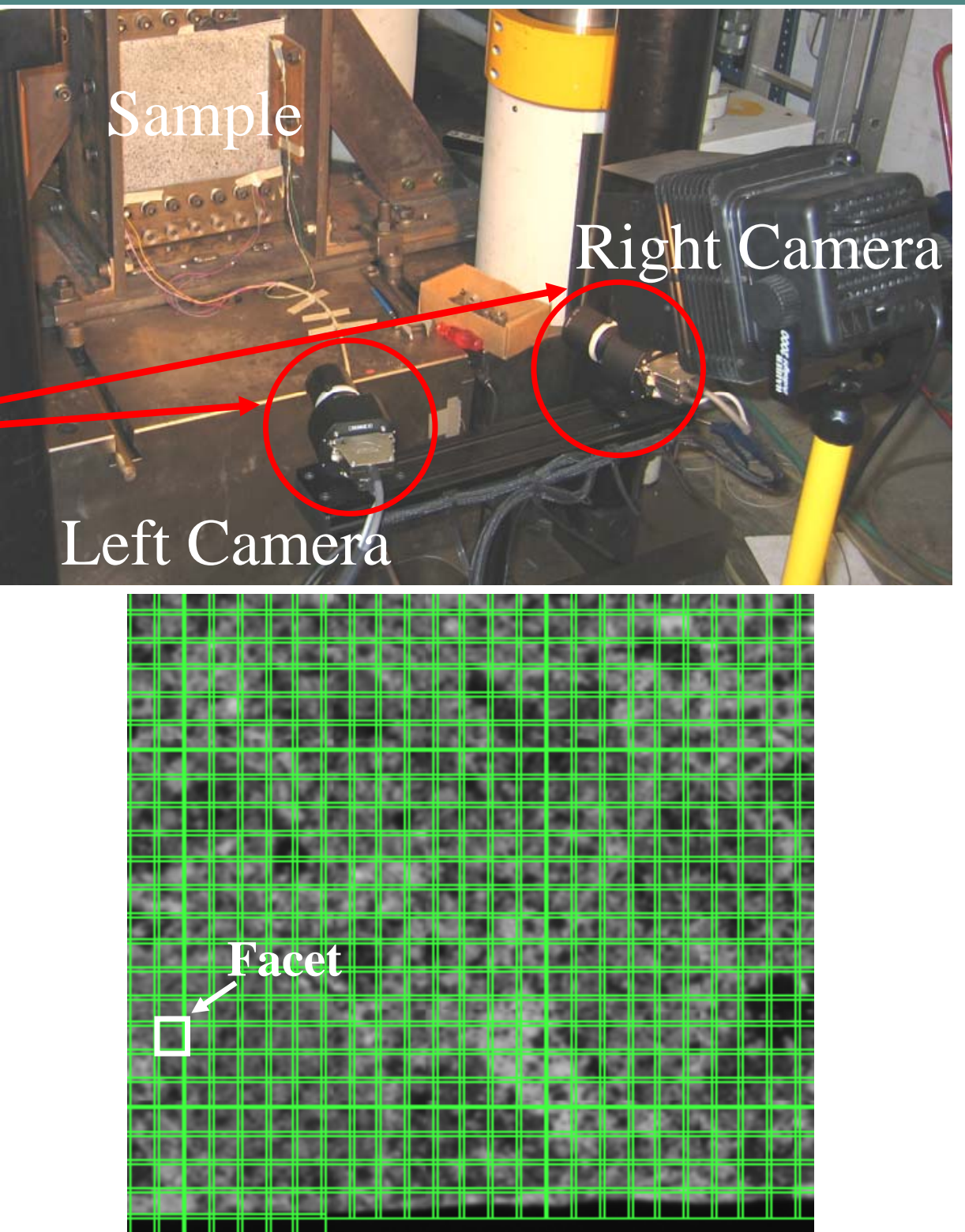
3-D Photogrammetry:

\section{General Measurement Process}

- Coordinates assigned to facet corner and center by grayscale variation

- System tracks movement of facet center and corners
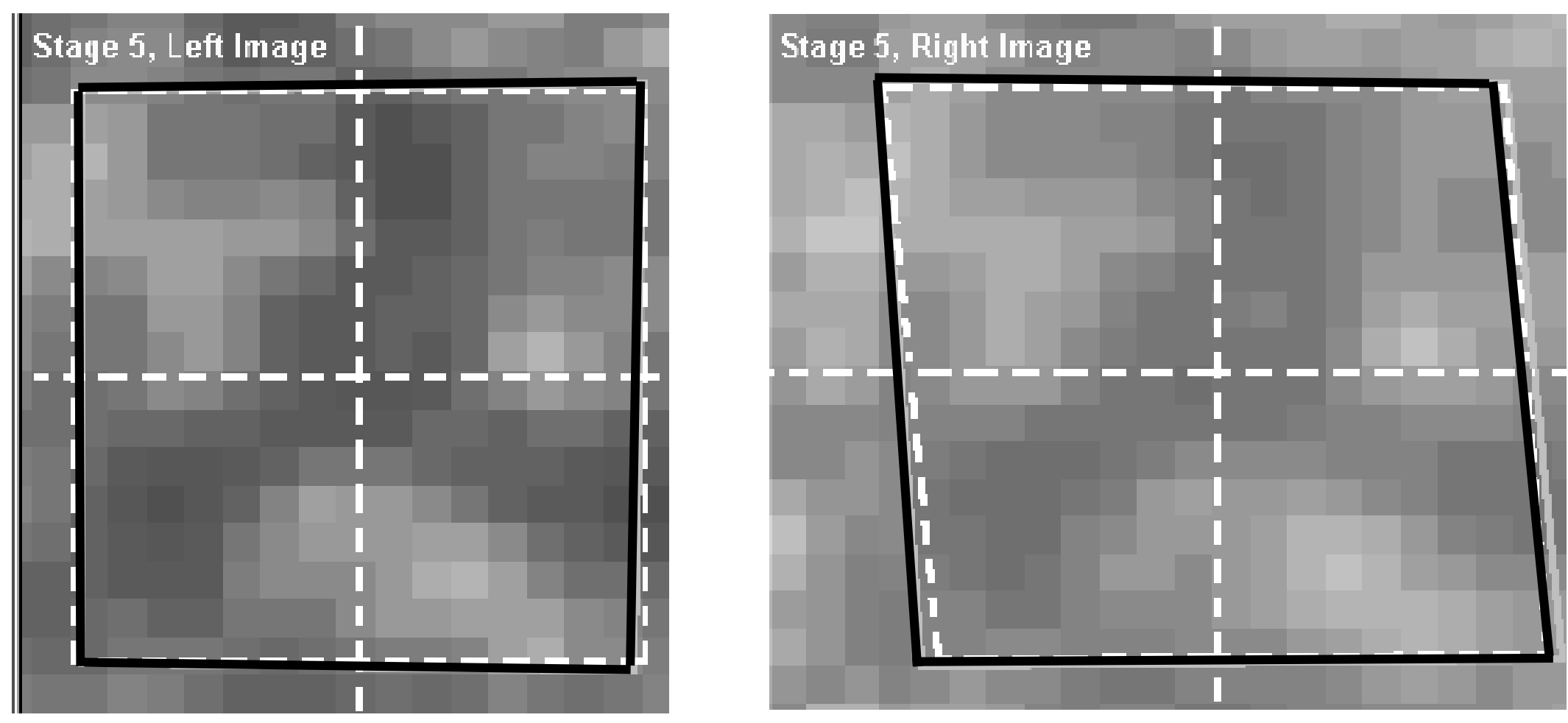

White line - original location

Black line - deformed shape 
Aggregate

Reinforcement

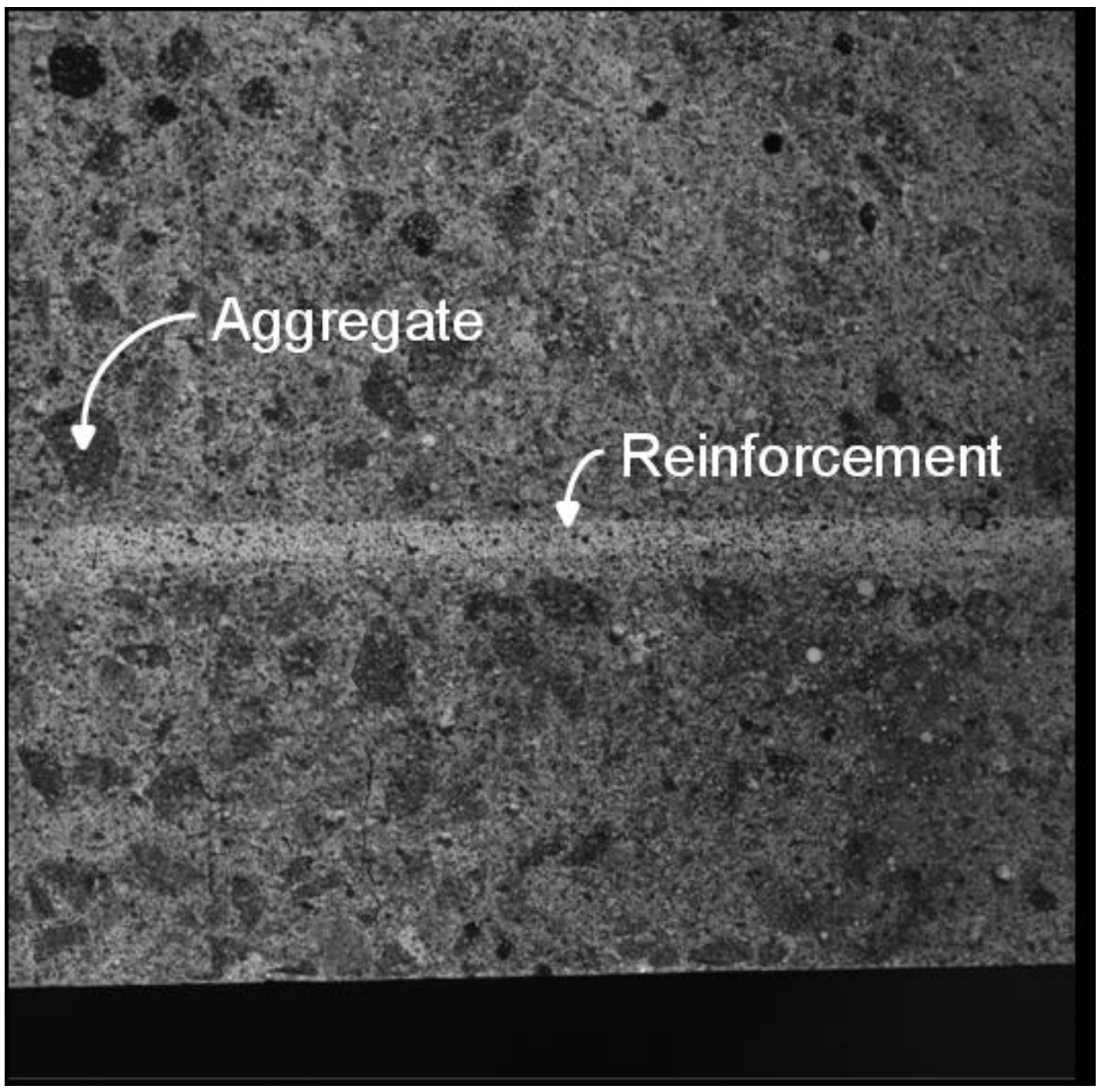

\section{3-D Photogrammetry: Surface Preparation

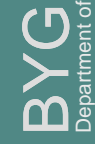 \\ - Grayscale contrast obtained by speckle pattern




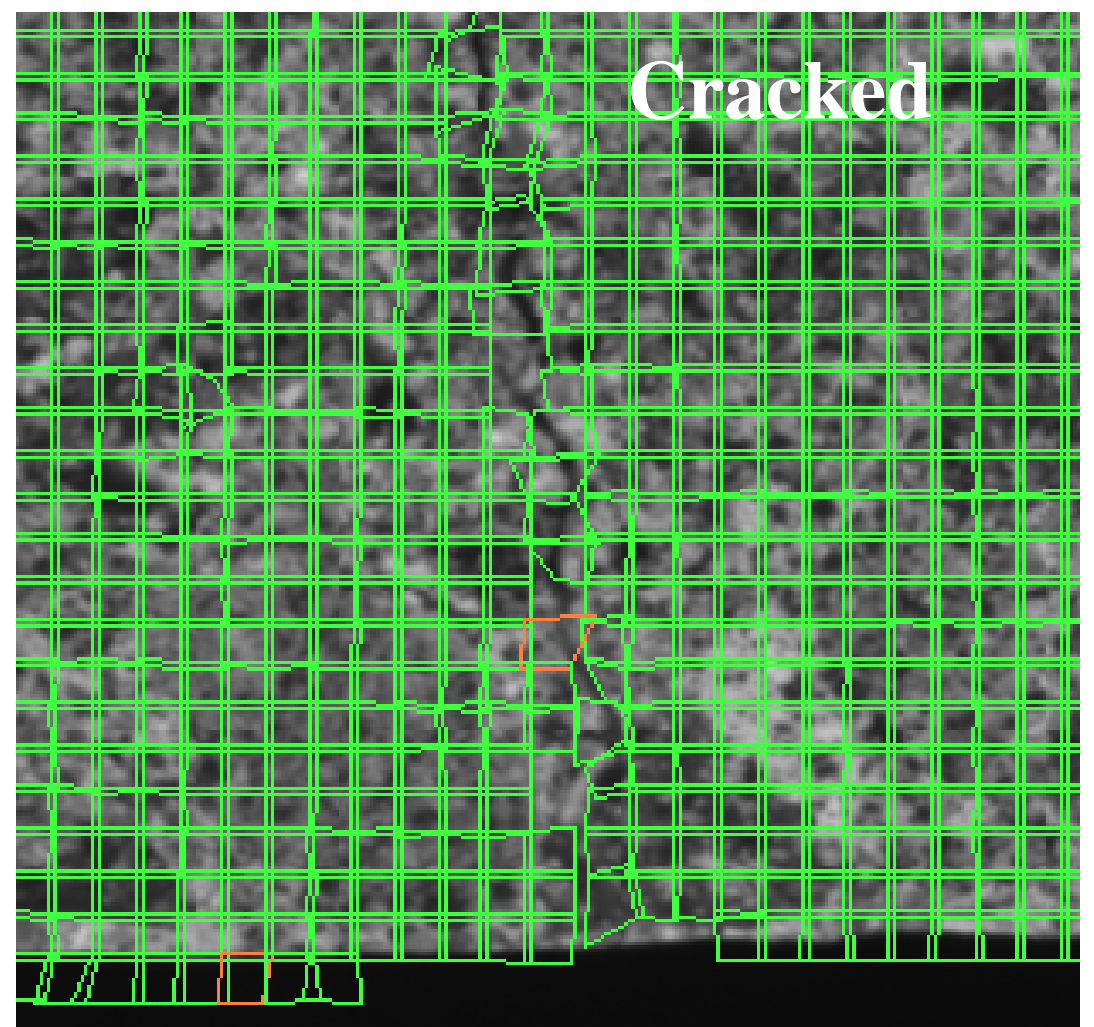

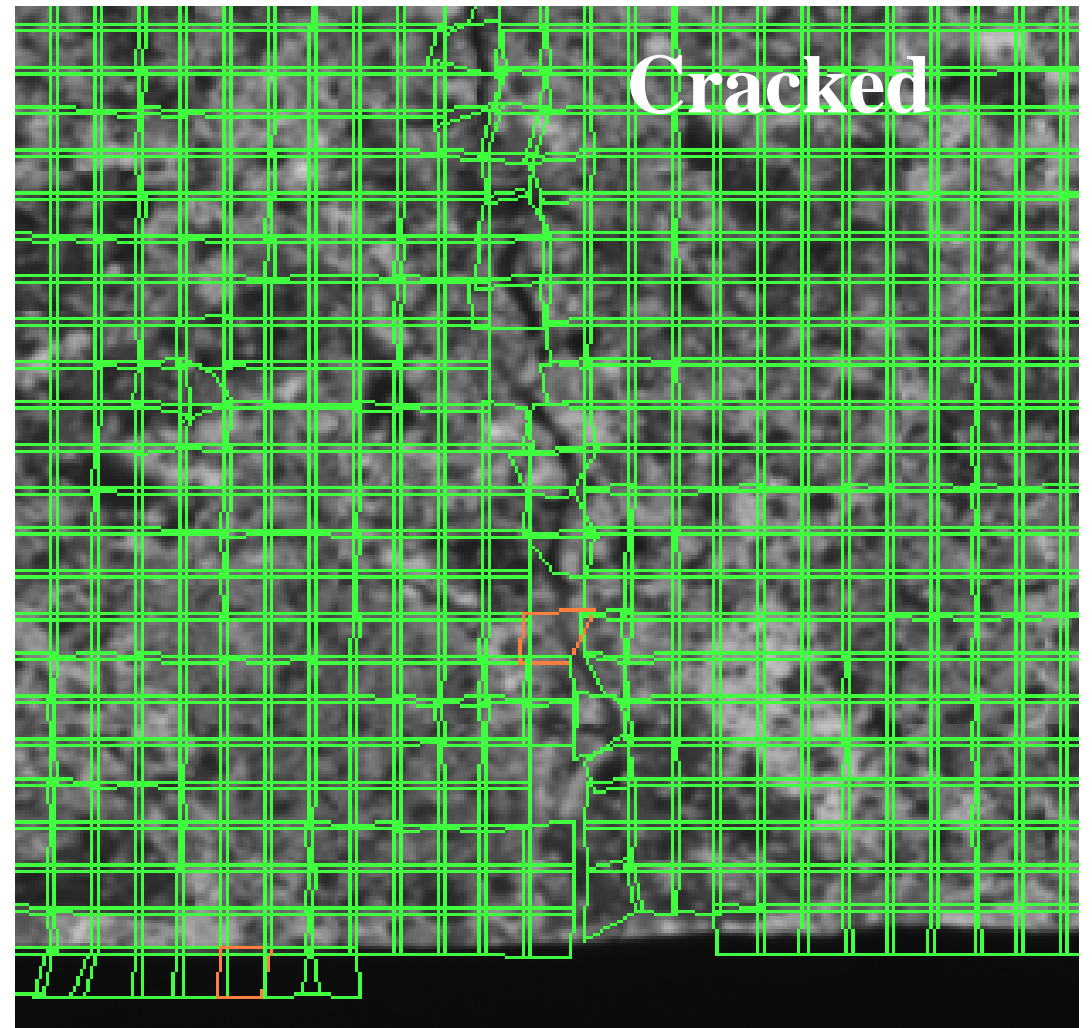

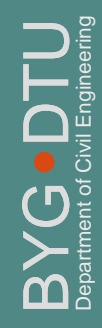 \\ 3-D Photogrammetry:
Deformation Measurement
deformation measured as mesh \\ 3-D Photogrammetry:
Deformation Measurement
deformation measured as mesh \\ 3-D Photogrammetry:
Deformation Measurement

\section{movements ,}

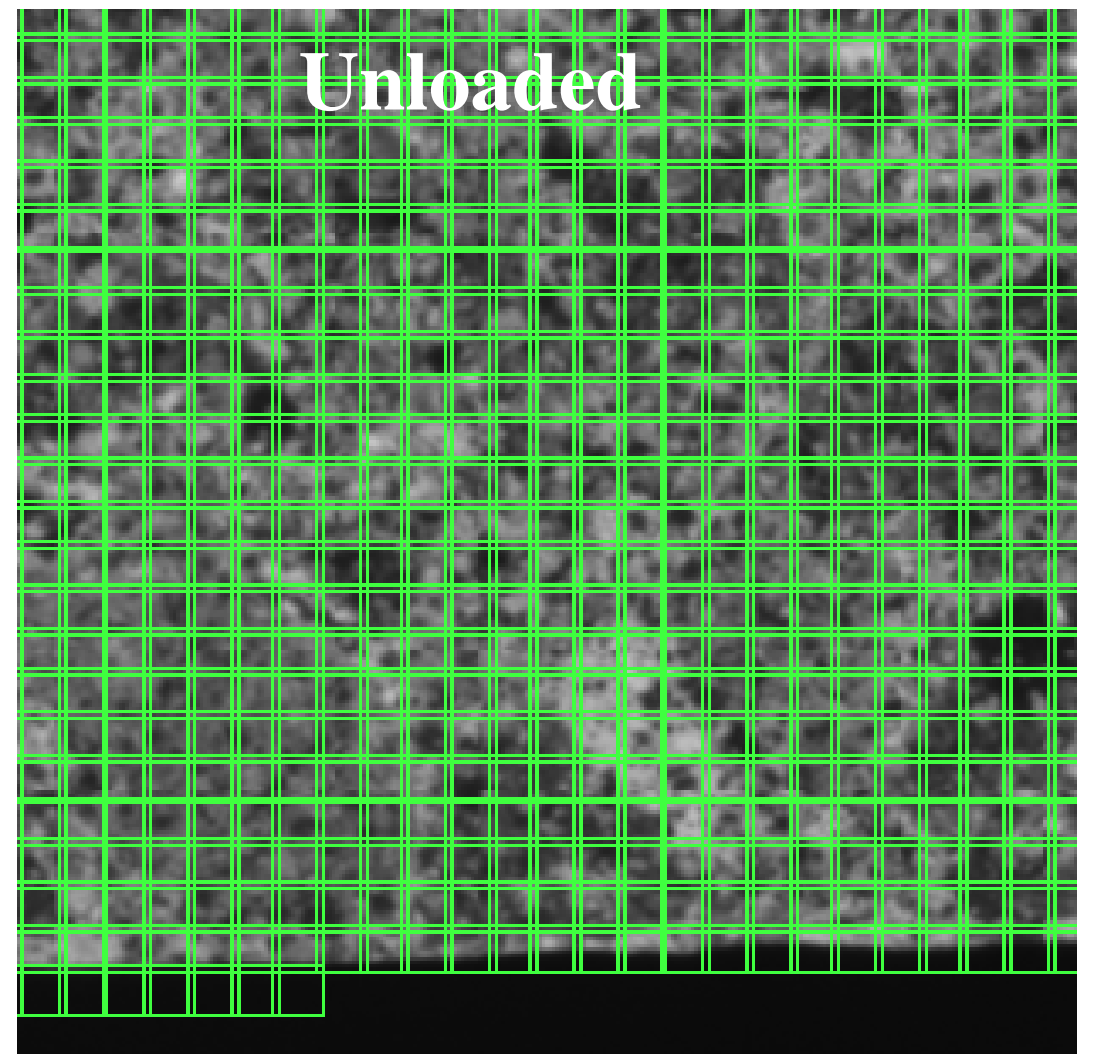

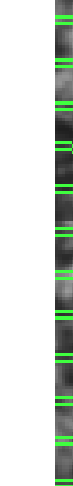




\section{Outline of Today's Talk}

- Motivation \& Goal
- Experimental Investigation
- Three-Dimensional Photogrammetry Technique

- Motivation \& Goal
- Experimental Investigation
- Three-Dimensional Photogrammetry Technique

- Motivation \& Goal
- Experimental Investigation
- Three-Dimensional Photogrammetry Technique

- Test Results
- Crack Geometry
- Crack Measurements

- Test Results
- Crack Geometry
- Crack Measurements

- Test Results
- Crack Geometry
- Crack Measurements

- Conclusions

(

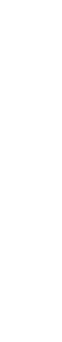

政

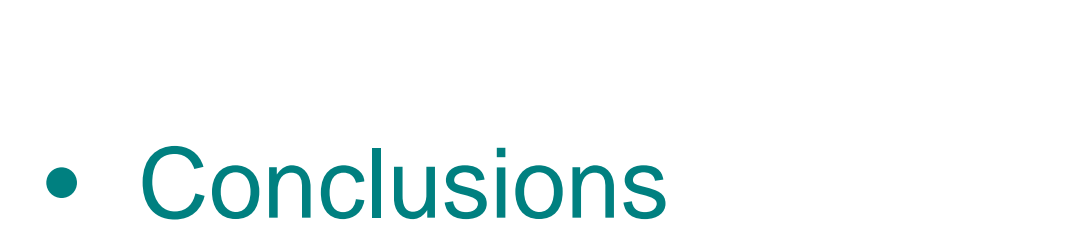

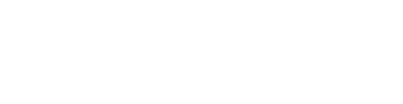

- Motivation \& Goal
- Experimental Investigation
- Three-Dimensional Photogrammetry Technique

Conclusions

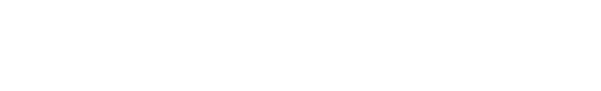

- Motivation \& Goal
- Experimental Investigation
- Three-Dimensional Photogrammetry Technique

- Motivation \& Goal
- Experimental Investigation
- Three-Dimensional Photogrammetry Technique

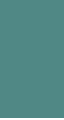

- Motivation \& Goal
- Experimental Investigation
- Three-Dimensional Photogrammetry Technique 


\section{3-D Photogrammetry: Crack Geometry}

- All beams loaded at constant rate, maximum - 3 min. unloaded $-3 \mathrm{~min}$.

- Video of measured deformation projected on beam surface

- Crack opening measurements from

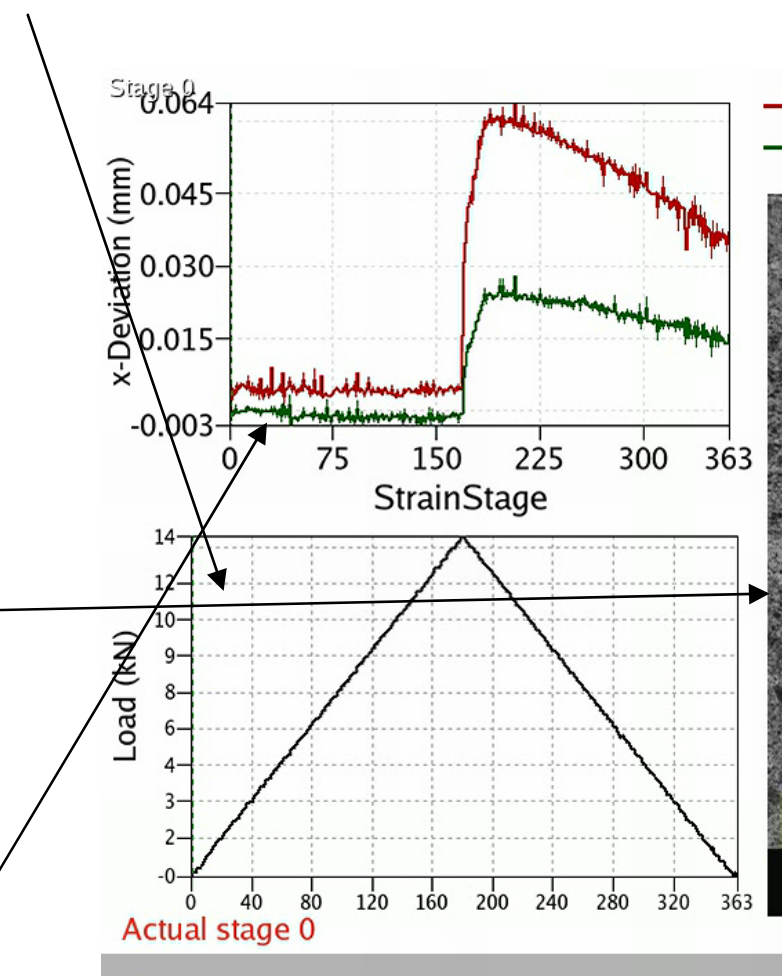

Crack Opening 1: X-Deviation Crack Opening 2: X-Deviation tension face and near reinforcement 


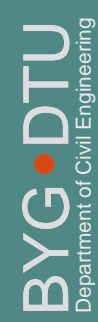

- Load vs. crack width at various

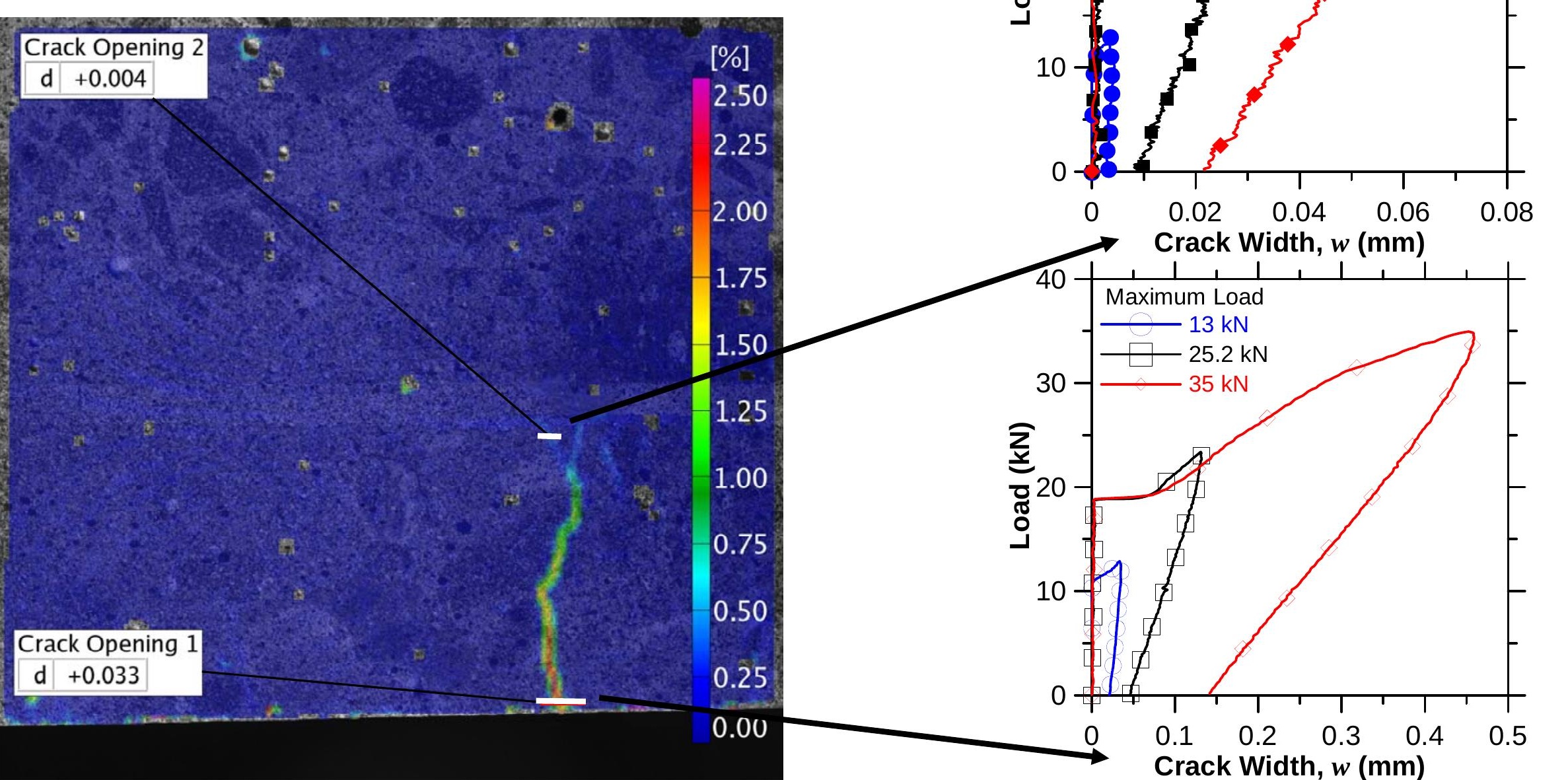

\section{Crack Measurements: Crack Shape}

$40 \int_{-\frac{1}{\text { Maximum Load }}}^{\frac{1}{-13 \mathrm{kN}}}$

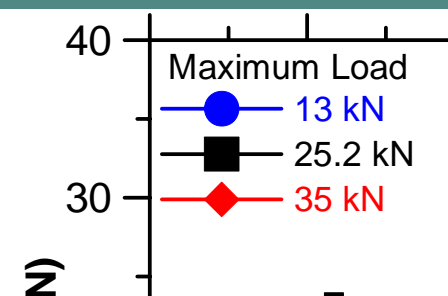

$30-35 \mathrm{kN}$

(6)

Crack Opening 2

d +0.004

Crack Opening 1

d +0.033 $\underset{z}{\underline{z}}$

20

ช్తి

.

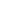

\section{loadings from two locations \\ - V-Shaped crack}
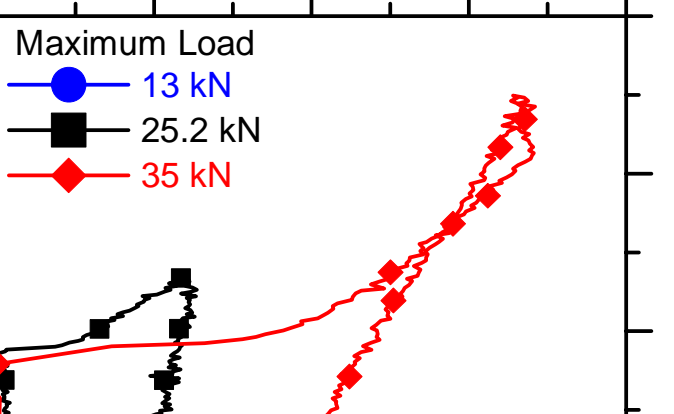


\section{Crack Measurements: Slip and Separation}

- Slip and separation vs. crack width near tensile face

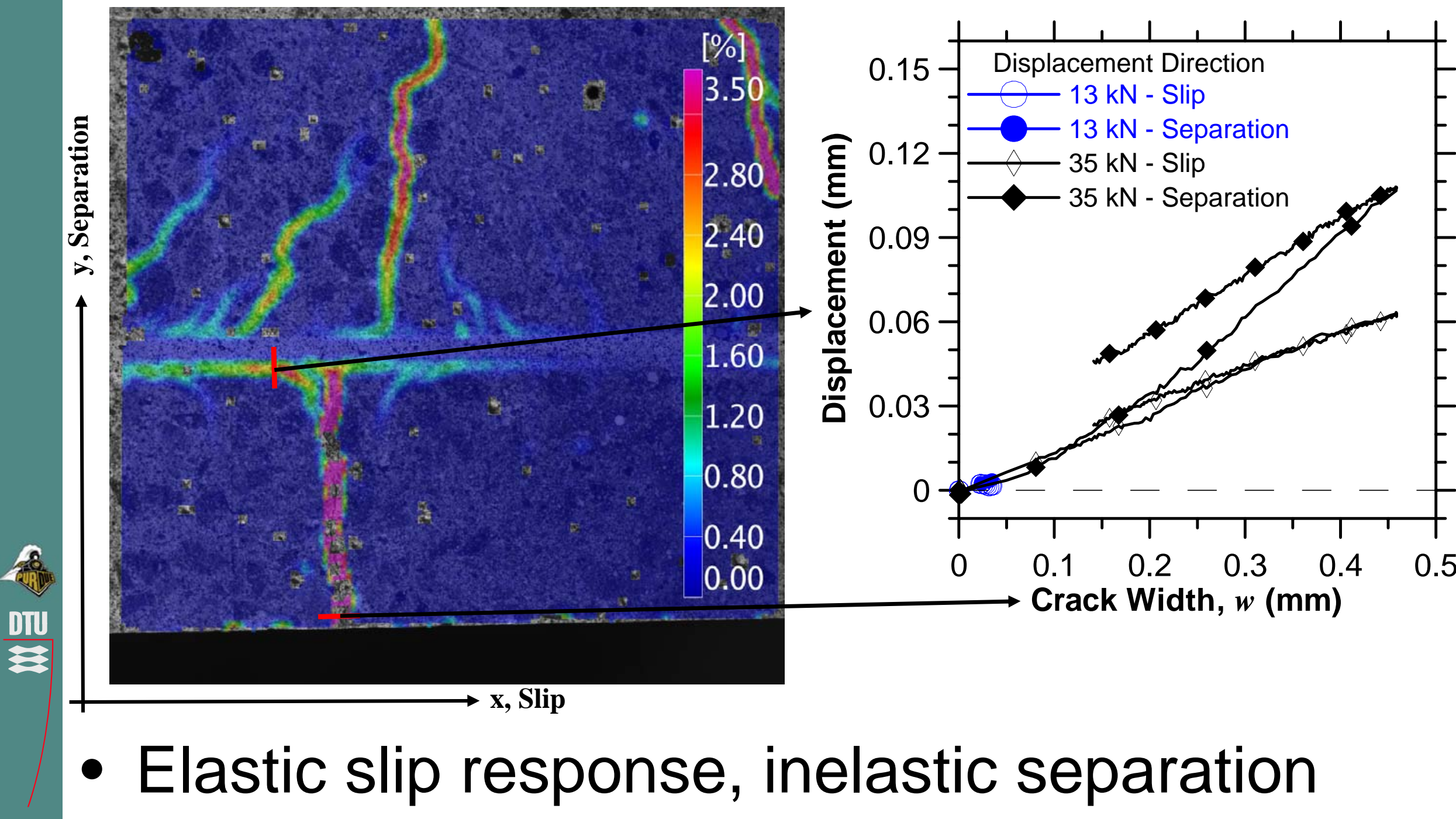

- Elastic slip response, inelastic separation 


\section{Crack Measurements: Slip and Separation}

- Initiation of slip and separation

- At estimated cracking load $\sim 4 \mu \mathrm{m}$ measured slip and separation

- Measured deformations close to resolution, results inconclusive

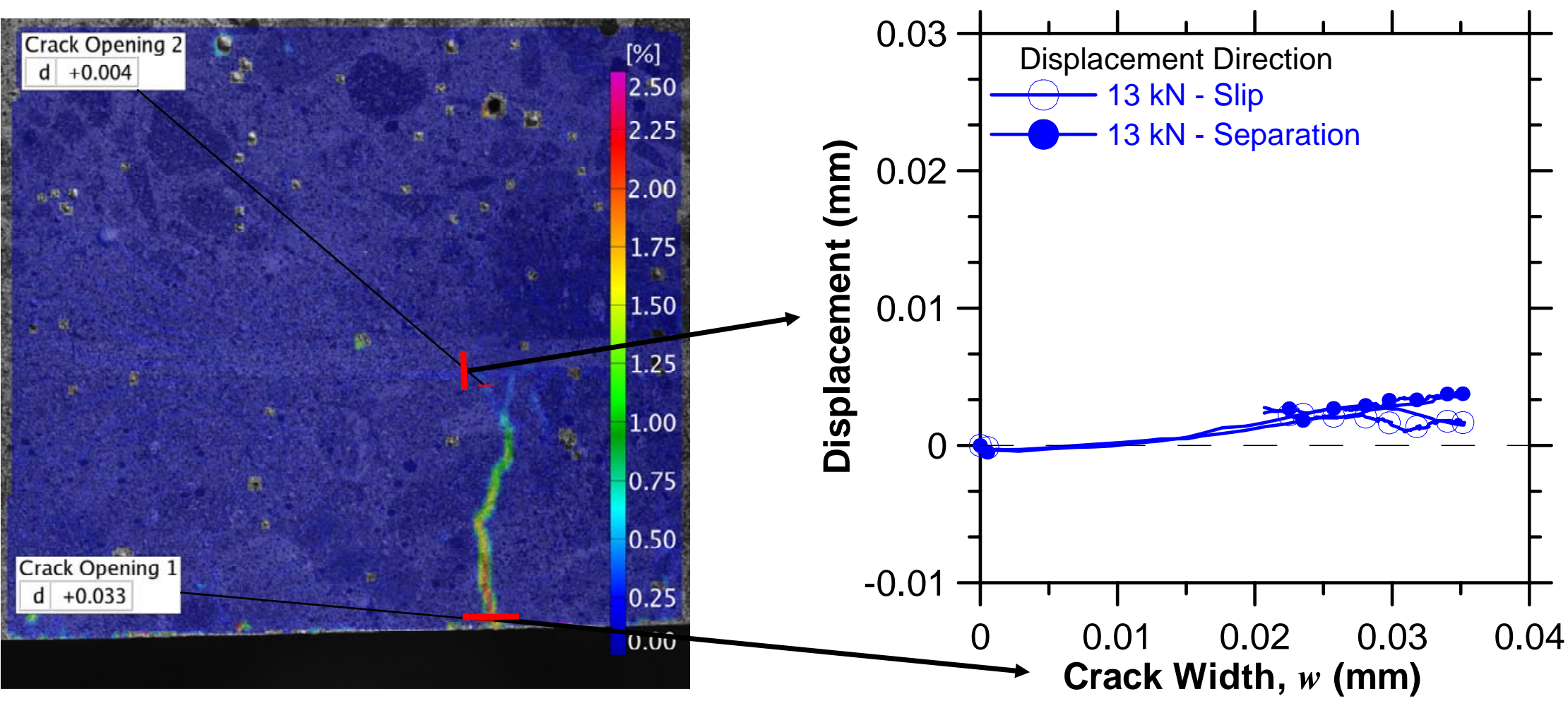




\section{Conclusions}

- 3-D Photogrammetry used to quantify cracking under flexural loading

- Slip behavior between reinforcement and concrete is elastic while separation behavior is inelastic

- Slip and separation between reinforcement and concrete initiates after opening of crack at tensile face 
